\title{
The Kähler Cone as Cosmic Censor
}

\author{
Christoph Mayer and Thomas Mohaupt \\ Theoretisch-Physikalisches Institut, Friedrich-Schiller-Universität Jena, \\ Max-Wien-Platz 1, D-07743 Jena, Germany \\ E-mail: C.Mayer@tpi.uni-jena.de, T.Mohaupt@tpi.uni-jena.de
}

\begin{abstract}
M-theory effects prevent five-dimensional domain-wall and black-hole solutions from developing curvature singularities. While so far this analysis was performed for particular models, we now present a model-independent proof that these solutions do not have naked singularities as long as the Kähler moduli take values inside the extended Kähler cone. As a by-product we obtain information on the regularity of the Kähler-cone metric at boundaries of the Kähler cone and derive relations between the geometry of moduli space and space-time.
\end{abstract}

Submitted to: Class. Quantum Grav.

PACS numbers: 04.20.Dw, 11.25.Yb, 04.65.+e, 11.27.+d, 04.70-s 


\section{Introduction}

Singularities appear quite generically in classical gravity [1]. The cosmic censorship conjecture states that the only singularities of physical space-times can be: an initial cosmological singularity (big bang), a final cosmological singularity (big crunch), and singularities resulting from gravitational collapse, which are hidden behind event horizons [2]. To be precise, this is just one version of the cosmic censorship conjecture, called "version 2, physical formulation" in [3], to which we refer for an overview. In the case of four-dimensional static space-times, supersymmetry acts as a cosmic censor [4], but, since there are non-static stationary supersymmetric solutions with naked singularities [5], it is clear that supersymmetry alone is not enough to establish cosmic censorship.

A satisfactory quantum theory of gravity should not only be capable of establishing cosmic censorship within the semi-classical approximation, but also be able to resolve the cosmological and black-hole singularities. String and M-theory are currently not able to achieve these goals in general, but have already provided a variety of insights into the problem of singularities. One new ingredient is the existence of a length scale, $\sqrt{\alpha^{\prime}}$, at which internal string states can be excited. This leads to a very soft UV behaviour of scattering amplitudes, which reflects itself in an infinite series of higher derivative terms, and in particular higher curvature terms, in the low-energy effective action. Although this suggests that curvature singularities are smoothed out, it is very hard to make this explicit, except in situations where the corresponding space-time can be described by an exact conformal field theory. See for example [6] for a recent application of such techniques to cosmology.

Besides higher derivative terms, there is another generic mechanism for avoiding singularities in string and M-theory, which one might call "the intervention of additional states." One example of this mechanism are twisted states in toroidal orbifold compactifications [7], which prevent the conic singularities of these spaces to cause singularities of observable quantities. Recently, time-dependent orbifolds [8] have become important as models for space-like singularities, including cosmological singularities [9, 10]. See [11] for a review and more references. More elaborate versions of the two basic mechanisms take care of the geometrical singularities occurring at special points in the moduli spaces of Calabi-Yau compactifications. Such special points are related to flop transitions [12], conifold singularities [13], conifold transitions [14] and more general extremal transitions [15, 16]. Here, in general, both $\alpha^{\prime}$-corrections and the presence of additional light states descending from p-branes wrapped on p-cycles of the internal manifold need to be taken into account in order to obtain non-singular physical quantities.

While the above examples concern singularities in an internal, compact manifold, one can also obtain new insights into space-time singularities by considering the 
full string or M-theory dynamics instead of a naive supergravity approximation. A fascinating interplay between internal, compact space and non-compact space-time is exhibited by the enhançon [17]. Here, one considers certain space-time geometries which have a naked curvature singularity in the supergravity approximation. However, by considering the full string theory one realises that before the singularity can be reached, particular modes of branes wrapped on internal cycles become light, and therefore must be taken into account. The resulting space-time geometry is then free of naked singularities. This mechanism, which has been first observed in a specific compactification with $\mathcal{N}=4$ supersymmetry, seems to be quite generic. For instance, a version of it was discovered in domain-wall solutions of eleven-dimensional supergravity compactified on a Calabi-Yau three-fold with G-flux 18. This compactification describes the bulk dynamics of five-dimensional heterotic M-theory 19, 20. Moreover, it was shown in 221] that a similar mechanism prohibits naked singularities of electric and magnetic BPS solutions of ungauged five-dimensional supergravity, when embedded into M-theory compactified on a Calabi-Yau three-fold (without G-flux). However, except in the case of magnetic BPS solutions, one has specific examples instead of a general proof.

In this paper we make a step towards a model-independent analysis of space-time singularities in M-theory compactifications. The basic idea is to work out systematically the relation between the geometries of the internal space and of space-time, and to prove that one always encounters new M-theory physics, such as additional light states, when, or even before a naked space-time singularity occurs. Since we work with the dimensionally reduced low-energy effective action, the geometry of the internal space is encoded in space-time dependent scalar fields (moduli), while the space-time geometry is obtained by solving the equations of motion. We therefore have to relate spacetime geometry, in particular curvature invariants to the geometry of the moduli space. Concretely, we consider eleven-dimensional supergravity compactified on Calabi-Yau three-folds and prove that five-dimensional BPS domain-wall solutions (and electric BPS solutions) cannot have curvature singularities as long as the moduli fields take values in the interior of the extended Kähler cone. The only way solutions can become singular is when the boundary of the extended Kähler cone is reached, but there the internal manifold becomes singular and the description in terms of a five-dimensional effective supergravity theory is not valid. We will not address the physics of these singularities in this paper, but make a proposal how they can be approached in the conclusions. There are also supergravity solutions with naked singularities which are not related to boundaries of the extended Kähler cone [18]. These are artifacts of a naive supergravity treatment. The extended Kähler cone is obtained by gluing together the Kähler cones of different Calabi-Yau spaces, which are related by topological transitions. Along the corresponding internal boundaries of the extended Kähler cone, the effective supergravity lagrangian is non-singular, but its parameters change through threshold 
corrections of M-theory modes which become massless. If this effect is ignored, one leaves the M-theory moduli space, and naked space-time singularities can and will occur. If, however, the effects of the interior boundaries are taken into account correctly, one moves from the Kähler cone of one Calabi-Yau three-fold into the Kähler cone of another one. In this paper we prove that solutions are non-singular while the moduli take values (i) inside the Kähler cone or (ii) on interior boundaries of the extended Kähler cone. Therefore we know that as long as the scalars are inside the extended Kähler cone no naked singularities occur. If they seem to be present in a naive supergravity treatment, we are guaranteed to reach an internal boundary of the extended Kähler cone before we reach the singularity.

In other words, the "enhançon-mechanism" for domain walls and electric BPS solutions, which was observed in particular models [18, 21], works in general. This implies that electric BPS solutions, which are the space-times of minimal ADM mass for a given total electric charge, are always black holes, and never have naked singularities, as long as the moduli take values inside the extended Kähler cone. Similarly, domain walls can only become singular when the moduli reach the boundary of the extended Kähler cone. Thus we establish an, albeit limited, version of cosmic censorship which might be phrased as "the Kähler cone is a cosmic censor." As a by-product we obtain various nice relations between geometrical quantities of the Kähler cone and of spacetime. We show explicitly how the behaviour of the metric of the Kähler cone on the boundaries is related to the geometrical degeneration and the new physics occurring there. Our results confirm that the interplay between internal and space-time geometry is of central importance in string theory. Moreover, when working with effective supergravity actions, string theory physics is only captured if all relevant modes of the full theory are taken into account. For more work along these lines see [21, 22, 23].

The outline of this paper is as follows. Section 2.1 introduces domain-wall solutions of five-dimensional gauged supergravity theories. The analysis of space-time curvature singularities is performed in section 2.2, and the relation to Calabi-Yau flux compactifications of M-theory is reviewed in section 2.3. The main results of this paper reside in section 3, where certain properties of the Calabi-Yau Kähler-cone metric are proven. In section 4 we explain how our arguments can be applied to electric BPS solutions of ungauged five-dimensional supergravity. Our conclusions are given in section 5 .

\section{BPS Domain-wall Solutions of Five-dimensional Gauged Supergravity}

We consider two classes of $1 / 2$-BPS solutions of five-dimensional $\mathcal{N}=2$ supergravity [24, 25, 26], domain-wall, and black-hole solutions. Since these solutions have many features in common, we focus on the domain-wall solutions in the following. The following arguments can be adapted to black-hole solutions, as we will show in section 4 . 


\subsection{Review of Domain-wall Solutions}

In this subsection, we review the domain-wall solution of a class of five-dimensional gauged supergravity theories, which describes the bulk dynamics of Hořava-Witten theory compactified on a Calabi-Yau three-fold [19, 20, 27, 28].

The bosonic fields are part of the following multiplets: Metric and graviphoton, $\left\{g_{\mu \nu}, A_{\mu}\right\}$, belong to the gravity multiplet. There are $N-1$ vector fields and scalars, $\left\{A_{\mu}^{i}, \phi^{i}\right\}, i \in 1 \ldots N-1$ in vector multiplets. Furthermore, we consider the universal hypermultiplet (UHM), $\{V, a, \xi, \bar{\xi}\}$, consisting of two real and one complex scalar. The theory might contain additional hypermultiplets which, however, do not play a role in the domain-wall solutions we consider.

The scalar fields $\phi^{i}$ parametrise a degree-three hyper-surface in $\mathbb{R}^{N}$ [24, 29]

$$
\mathcal{V}(X):=\frac{1}{6} c_{I J K} X^{I} X^{J} X^{K}=1, \quad I, J, K \in 1 \ldots N,
$$

determined by the real, symmetric, and constant coefficients $c_{I J K}$. As the graviphoton $A$ and the vector multiplet gauge fields $A^{i}$ combine into $N$ vector fields $A^{I}$, we combine the $N-1$ scalars $\phi^{i}$ and $V$ together, anticipating the structure we will obtain by dimensional reduction, and define

$$
Y^{I}:=V^{1 / 6} X^{I}
$$

We consider a particular gauging of the axion in the UHM, which induces a potential for the moduli. As a consequence, neither flat Minkowski space nor $A d S_{5}$ space is a solution. The most symmetric solutions are 1/2-BPS solutions, invariant under 4 supercharges and under 4-dimensional Lorentz transformations, only.

The five-dimensional line element of such a domain-wall solution is given by [19, 20]

$\mathrm{d} s^{2}=\exp [2 U(y)]\left\{-\left(\mathrm{d} x^{0}\right)^{2}+\left(\mathrm{d} x^{1}\right)^{2}+\left(\mathrm{d} x^{2}\right)^{2}+\left(\mathrm{d} x^{3}\right)^{2}\right\}+\exp [8 U(y)] \mathrm{d} y^{2}$,

in terms of a single function $U$, which only depends on the transversal coordinate $y$. This function is related to the scalar moduli by

$$
\exp [6 U(y)]=V(y)=\left(\frac{1}{6} c_{I J K} Y^{I}(y) Y^{J}(y) Y^{K}(y)\right)^{2} .
$$

The moduli $Y^{I}(y)$, in turn, are determined in terms of harmonic functions $H_{I}(y)$,

$$
c_{I J K} Y^{I}(y) Y^{K}(y)=2 H_{I}(y), \quad H_{I}(y)=a_{I} y+b_{I}, \quad a_{I}, b_{I} \in \mathbb{R} .
$$

Note that the domain-wall solution is completely fixed by a flow on the scalar manifold which is parameterised by the transverse coordinate $y$. The solution starts at $y=y_{1}$ at a particular point on the scalar manifold and evolves as determined by the equations (2.3)(2.5) until it terminates at a different point at $y=y_{2}$. Since the five-dimensional theory does not have fully supersymmetric ground states, there is no fixed-point behaviour and we have to introduce boundaries at the positions $y_{1}, y_{2}$ by hand. The so-called 
generalised stabilisation equations (2.5) are an universal feature of both, domain-wall and black-hole solutions, and therefore the following analysis of (space-time) curvature singularities can be adapted for black-hole solutions, as we will show in section 4 .

\subsection{Curvature Singularities of Domain-wall Solutions}

Here, we investigate the occurrence of space-time curvature singularities. We start by calculating the Ricci scalar of the metric (2.3) and then analyse possible sources of divergences. The Ricci scalar is given by $\left({ }^{\prime}=\frac{\mathrm{d}}{\mathrm{d} y}\right)$

$$
R=4 \exp [-8 U]\left(3 U^{\prime} U^{\prime}-2 U^{\prime \prime}\right) \text {. }
$$

This expression can diverge ( $U$ is related to $V$ by equation (2.4)):

(i) if either $\exp [-U] \rightarrow \infty(V \rightarrow 0)$,

(ii) or if the first or second derivatives of $U$ (or $V$ ) diverge.

Since the line element (2.3) depends only on the function $U$, all components of the Riemann tensor are polynomials in $U^{\prime}$ and $U^{\prime \prime}$. Hence, our analysis applies to all curvature invariants of the domain-wall metric.

Since case (i) has already been covered in the literature 40, it remains to analyse the somewhat less obvious case (ii), i.e., diverging curvature invariants at finite and non-zero $V$. It is convenient to consider the first derivative of $\sqrt{V}$ instead of $V$ :

$$
(\sqrt{V})^{\prime}=\frac{1}{2} c_{I J K} Y^{I} Y^{J} Y^{\prime K}=\frac{1}{2} Y^{I} H_{I}^{\prime},
$$

where in the last step we have used the relation

$$
c_{I J K} Y^{J} Y^{\prime K}=H_{I}^{\prime},
$$

which follows from differentiating (2.5) with respect to $y$. Since the harmonic functions $H_{I}$ are at most linear in $y, V^{\prime}$ is regular as long as the moduli $Y^{I}$ are finite [30, 18.

Differentiating equation (2.7) once more we find

$$
(\sqrt{V})^{\prime \prime}=\frac{1}{2} Y^{I I} H_{I}^{\prime}+\frac{1}{2} Y^{I} H_{I}^{\prime \prime}
$$

Clearly, $(\sqrt{V})^{\prime \prime}$ can blow up if $Y^{\prime I}$ diverges. By introducing the matrix 28

$$
\tilde{M}_{I J}=\frac{1}{2} c_{I J K} Y^{K}
$$

we can invert equation (2.8):

$$
Y^{\prime I}=\frac{1}{2} \tilde{M}^{I K} H_{K}^{\prime}
$$

Of course this inversion is only formal, because it requires to compute $\tilde{M}^{I J}$ as a function of the moduli $Y^{I}$. Since $H_{I}^{\prime}=a_{I}=$ const, $\left|Y^{I}\right| \rightarrow \infty$ when $\tilde{M}_{I J}$ is not invertible, or, equivalently, when $\operatorname{det} \tilde{M}=0$. Using the last equation, we obtain

$$
(\sqrt{V})^{\prime \prime}=\frac{1}{4} H_{I}^{\prime} \tilde{M}^{I J} H_{J}^{\prime}+\frac{1}{2} Y^{I} H_{I}^{\prime \prime}
$$


The appearance of the matrix $\tilde{M}_{I J}$ is the link between space-time curvature singularities and properties of the moduli-space metric, which we will deal with in section 3 .

We have shown that there are two possible causes for curvature singularities of domain-wall solutions: (i) $V \rightarrow 0$, and (ii) $\tilde{M}_{I J}$ non-invertible at finite $V \neq 0$. Let us demonstrate that these curvature singularities do occur generically: Since $V$ is a homogeneous function of degree three in the moduli $Y^{I}$, it will have zeros if the moduli $Y^{I}$ are allowed to take arbitrary real values. This covers case (i). As for case (ii), a generic matrix $\tilde{M}_{I J}$ is invertible, but becomes singular in co-dimension one in parameter space. In other words, if no additional conditions on the parameters are imposed, the set of solutions will decompose into two subsets: those, which do not cross the hyperplanes where $\tilde{M}_{I J}$ becomes singular, and those which do. This can be seen explicitly in the examples considered in Ref. [18]. In both cases singular and non-singular spacetime geometries are equally generic, and supergravity does not provide any constraints on the parameters which exclude the singular solutions. The difference between case (i) and case (ii) is that in the first case the metric on the scalar manifold diverges, while it develops a zero eigenvalue in the second case. Thus, the five-dimensional supergravity lagrangian becomes singular, which indicates that we need input from an underlying fundamental theory. We will see that this input is provided by M-theory, if the supergravity theory is obtained as a Calabi-Yau compactification.

\subsection{Compactification of Eleven-dimensional Supergravity on Calabi-Yau Three-folds with Background Flux}

Here, we recall how five-dimensional gauged supergravity can be obtained by compactification of eleven-dimensional supergravity on Calabi-Yau three-folds in the presence of background flux.

There are two points of view concerning the relation of gauged five-dimensional supergravity actions to eleven-dimensional supergravity theory: (i) compactification on

a Calabi-Yau manifold, assuming that the flux only excites Calabi-Yau zero-modes and does not deform the Calabi-Yau structure. The presence of background flux is taken into account by including it as a "non-zero mode," see Refs. [19, 20, 27]. Or, (ii), compactification on a "deformed" Calabi-Yau manifold [19, 20, 31, 32.

We describe the first approach. The bosonic fields of eleven-dimensional supergravity consist of the metric and of a three-form gauge potential $C_{3}$ with associated four-form field strength $G_{4}=\mathrm{d} C_{3}$. We start by specifying a basis of the second cohomology group consisting of $h^{1,1}$ harmonic $(1,1)$ forms $\omega_{I}$. The Kähler form can be expanded in this basis,

$$
J=\mathcal{Y}^{I} \omega_{I}, \quad\left\langle\omega_{I}\right\rangle=H^{1,1}(X), \quad I=1 \ldots h^{1,1}:=\operatorname{dim} H^{1,1}(X),
$$

with real moduli $\mathcal{Y}^{I}$, which are related to the moduli of secion 2 by the rescaling $\mathcal{Y}^{I}:=V^{1 / 3} X^{I}=V^{1 / 6} Y^{I}$. Since we will need a basis of $H^{2,2}(X)$ and of the even homology 
of $X$, we introduce dual 4-forms, 2-cycles, and 4-cycles. By Poincaré duality, there is a dual basis of 4 -forms $\nu^{I}$ defined as

$$
\int_{X} \nu^{I} \wedge \omega_{J}=\delta_{J}^{I}, \quad\left\langle\nu^{I}\right\rangle=H^{2,2}(X)
$$

In homology, we fix a basis of 2- and 4-cycles, with relations

$$
\int_{C^{I}} \omega_{J}=\int_{D_{J}} \nu^{I}=\delta_{J}^{I}, \quad\left\langle C^{I}\right\rangle=H_{2}(X), \quad\left\langle D_{I}\right\rangle=H_{4}(X) .
$$

The symmetric tensor $c_{I J K}$ of section 2 acquires now the interpretation of tripleintersection numbers

$$
c_{I J K}=D_{I} \circ D_{J} \circ D_{K}=\int_{X} \omega_{I} \wedge \omega_{J} \wedge \omega_{K}
$$

which implies that it is integer valued, $\ddagger$ in contrast to pure five-dimensional supergravity where real-valued tensors are allowed.

Having introduced a basis for the even (co-)homology, we now describe how the bosonic fields of section 2 descend from the fields of eleven-dimensional supergravity:

$$
\begin{array}{ll}
C_{M N P} & \Longrightarrow A_{\mu}^{I} \mathrm{~d} x^{\mu} \wedge \omega_{I}, \quad \xi \Omega_{a b c}, \quad \bar{\xi} \bar{\Omega}_{\bar{a} \bar{b} \bar{c}} \\
G_{M N P Q}=(\mathrm{d} C)_{M N P Q} & \Longrightarrow \mathrm{d} a=\star_{5} G \\
g_{M N} & \Longrightarrow \mathcal{Y}^{I} \omega_{I}, \quad g_{\mu \nu}
\end{array}
$$

Here, $\Omega_{a b c}$ denotes the holomorphic $(3,0)$ form which exists on every Calabi-Yau threefold. The fields $X^{I}$ of section 2.1 parameterise the relative sizes of the cycles of $X$, whereas the UHM scalar $V$ parameterises the volume of $X$. The axion $a$ comes from dualising the dimensionally reduced 4-form field strength, and therefore has a shift symmetry: $a \rightarrow a+c$.

In principle, dimensional reduction on a generic Calabi-Yau manifold yields more hypermultiplets than the UHM alone. For the type of domain-wall solutions we consider, these extra hypermultiplets are spectators, and it is a consistent truncation to keep these fields constant.

Following [19, 20, 27] we turn on background flux in a way that the flux backreaction on geometry is such that it excites Calabi-Yau zero-modes only, and does not distort the Calabi-Yau structure. Since the background four-form flux is an element of $H^{4}(X)=H^{2,2}(X)$, it can be expanded as follows

$$
G=\alpha_{I} \nu^{I} \in H^{2,2}(X)
$$

with constants $\alpha_{I}$ subject to a quantisation condition [33. However, within the supergravity approximation the flux parameters can be taken to be continuous as discussed in Ref. [32]. In the dimensionally reduced five-dimensional theory, turning $\ddagger$ This holds in an appropriate basis of (co-)homology and for non-singular X. For singular Calabi-Yau three-folds these numbers can be rational. 
on flux (2.13) leads to (i) a potential for the moduli $\mathcal{Y}^{I}$ and (ii) gauging of the shift symmetry of the axion, $D_{\mu} a=\partial_{\mu} a+\alpha_{I} A_{\mu}^{I}$.

It is important to keep in mind that the domain-wall solutions of the last subsections are exact solutions of five-dimensional gauged supergravity theory, but do not lift to exact solutions of the eleven dimensional theory. The corresponding eleven-dimensional domain-wall solutions of Hořava-Witten theory are only known up to first order, and to this order they agree with the five-dimensional domain-wall solutions, see Ref. [20].

We have already mentioned that the tensor $c_{I J K}$ has to be integer valued in a CalabiYau compactification. Similarly, the scalar fields $\mathcal{Y}^{I}$ are subject to certain constraints we will deal with in the next section.

\section{Properties of the Kähler Cone}

Having described five-dimensional domain-wall solutions from the point of view of supergravity in the last section, we now investigate the interplay between space-time physics and properties of the Kähler moduli space.

\subsection{The Kähler Cone of Calabi-Yau Three-folds}

By Wirtinger's theorem, the Kähler form measures the volume of holomorphic curves, surfaces and the volume of the Calabi-Yau manifold $X$. For all holomorphic curves $C \subset X$ and surfaces $S \subset X$, the following inequalities define the Kähler cone $\mathcal{K}$ :

$$
\begin{aligned}
& \operatorname{Vol}(C) \quad=\int_{C} J>0, \\
& \operatorname{Vol}(S) \quad=\frac{1}{2 !} \int_{S} J \wedge J>0, \\
& V:=\operatorname{Vol}(X)=\frac{1}{3 !} \int_{X} J \wedge J \wedge J=\frac{1}{6} c_{I J K} \mathcal{Y}^{I} \mathcal{Y}^{J} \mathcal{Y}^{K}=\mathcal{V}(\mathcal{Y})>0 .
\end{aligned}
$$

Thus, the Kähler moduli space has the structure of a cone. The (closure of) the Kähler cone is the cone $\overline{N E}^{1}(X)$ of nef classes, which is dual to (the closure of) the KleimanMori cone $\overline{N E}_{1}(X)$ of effective 2-cycles [34, 35]. The duality is given by the pairing $\operatorname{Pic}(X) \times H_{2}(X) \longrightarrow \mathbb{Z}$, which is $\int_{C} L$ for a curve $C$ and $L \in \operatorname{Pic}(X)=H^{1,1} \cap H^{2}(X)$, where $\operatorname{Pic}(X)$ denotes the Picard group of $X$. If $X$ is a Calabi-Yau three-fold, then the Kähler cone is locally polyhedral away from the so-called cubic cone $W:=\left\{\mathcal{Y}^{I} \in\right.$ $\mathbb{R} \mid V=0\}$ [34]. For toric-projective Calabi-Yau varieties the Kähler moduli space is a strongly convex finite polyhedral cone [36, 37], and there is an explicit, global parameterisation, which takes the form

$$
\mathcal{K}:=\left\{\mathcal{Y}^{I} \in \mathbb{R} \mid 0<\mathcal{Y}^{I}<\infty, 1 \leq I \leq h^{1,1}\right\} .
$$

We call this parameterisation adapted, since the moduli $\mathcal{Y}^{I}$ measure volumes of holomorphic 2-cycles $C_{I}$. 
The metric on the Calabi-Yau Kähler moduli space is given by 38, 39]

$$
G_{I J}:=\frac{1}{2 V} \int_{X} \omega_{I} \wedge \star \omega_{J}=-\frac{1}{2} \frac{\partial}{\partial \mathcal{Y}^{I}} \frac{\partial}{\partial \mathcal{Y}^{J}} \log \mathcal{V}(\mathcal{Y})
$$

This metric is non-degenerate inside the Kähler cone. With the use of equation (3.1) it can be rewritten as

$$
G_{I J}=-\frac{1}{V} M_{I K}\left(\delta_{J}^{K}-\frac{3}{2} T_{J}^{K}\right), \quad T_{J}^{K}:=\frac{1}{6 V} c_{J M N} \mathcal{Y}^{M} \mathcal{Y}^{N} \mathcal{Y}^{K} .
$$

Here, $M_{I J}=V^{1 / 6} \tilde{M}_{I J}$ is a rescaled version of the matrix $\tilde{M}_{I J}$ introduced in (2.9). The matrix $T$ is a projector, $T^{2}=T$, of trace one. By the Hodge index theorem, the signature of the matrix

$$
M_{I J}=\frac{1}{2} \int_{X} J \wedge \omega_{I} \wedge \omega_{J}=\frac{1}{2} c_{I J K} \mathcal{Y}^{K}
$$

is $\left(1, h^{1,1}-1\right)$ [34]. Since non-invertability of the matrix $M$ is one cause of space-time curvature singularities (see section 2.2), equation (3.4) establishes the link between the occurrence of curvature singularities and properties of the Kähler-cone metric. This connection can be made more explicit by calculating the determinant of $G$ :

$$
\operatorname{det} G=\left(\frac{-1}{V}\right)^{h^{1,1}} \operatorname{det} M \operatorname{det}\left(\mathbb{1}-\frac{3}{2} T\right)=-\frac{1}{2}\left(\frac{-1}{V}\right)^{h^{1,1}} \operatorname{det} M \text {, }
$$

where in the last step we have made use of the fact that $T$ is a projector of trace one. There is a basis in which $T$ assumes the form $T=\operatorname{diag}(1,0, \ldots 0)$, and we obtain

$$
\operatorname{det}\left(\mathbb{1}-\frac{3}{2} T\right)=\operatorname{det} \operatorname{diag}(-1 / 2,1, \ldots, 1)=-1 / 2,
$$

which completes the derivation of equation (3.5).

It is the aim of the next subsection to use the relation (3.5) in order to analyse regularity properties of the metric (3.3).

\subsection{Degenerations of the Kähler-cone Metric and Singularities of Space-time}

In this subsection, we analyse how the Kähler-cone metric (3.3) behaves on boundaries of the Kähler cone, in particular whether it develops zero eigenvalues. By "Kählercone metric at the boundary" we always mean the limit of the Kähler-cone metric as one approaches the boundary, and not the scalar metric of the extended effective field theories which explicitly include the additional light modes [40, 22, 23].

We consider boundaries of the Kähler cone where one particular 2-cycle, $C^{\star}$, collapses:

$$
\partial_{\star} \mathcal{K}:=\left\{\left(\mathcal{Y}^{\tilde{I}} \neq 0, \mathcal{Y}^{\star}=0\right), \quad 0<\mathcal{V}(\mathcal{Y})<\infty\right\}, \quad \tilde{I} \neq \star .
$$

The contractions at these co-dimension-one faces are called primitive. In Calabi-Yau three-folds the following contractions can take place [34, 40]: 
- Type I ("2 $\rightarrow 0$ "): A finite number of isolated curves in the homology class $C^{\star}$ is blown down to a set of points, $\operatorname{Vol}\left(C^{\star}\right)=\mathcal{Y}^{\star} \rightarrow 0$, e.g., (locally) $\mathcal{O}(-1) \oplus \mathcal{O}(-1) \longrightarrow$ $\mathbb{P}^{1}$.

- Type II (" $4 \rightarrow 0$ "): A divisor $D=v^{I} D_{I}$ collapses to a set of points: $\operatorname{Vol}(D) \propto\left(\mathcal{Y}^{\star}\right)^{2}$.

- Type III ("4 $\rightarrow 2$ "): A (complex) one-dimensional family of curves sweeps out a divisor $D=v^{I} D_{I}$. Contracting this family of curves induces a collapse of $D$ to a curve of genus $g$ : $\operatorname{Vol}(D) \propto \mathcal{Y}^{\star}$, e.g., $(g=0$ case $) \mathcal{O}(0) \oplus \mathcal{O}(-2) \longrightarrow \mathbb{P}^{1}$.

- Cubic cone ("6 $\rightarrow 4$ ", "6 $\rightarrow 2$ ", "6 $\rightarrow 0$ "): These contractions correspond to $V \propto \mathcal{Y}^{\star}, V \propto\left(\mathcal{Y}^{\star}\right)^{2}$ and $V \propto\left(\mathcal{Y}^{\star}\right)^{3}$.

Note that our definition of boundaries $\partial_{\star} \mathcal{K}$ in equation (3.6) does not include the last of these possibilities. This is already covered by case (i) in section 2.2: the Ricci scalar $\left(R \propto V^{-8 / 3}\right)$ diverges when $V \rightarrow 0$.

Boundaries of type I and type III can be crossed into the Kähler cone of a new Calabi-Yau threefold, which is birationally (and, for type III, even biholomorphically) equivalent to the original one. Crossing these boundaries corresponds to a flop [12] or going through gauge symmetry enhancement [34, 15], respectively. The extended Kähler cone is gotten by enlarging the Kähler moduli space at all boundaries of type I. Enlarging in addition the Kähler moduli space at all boundaries of type III, one obtains the extended movable cone [12, 15]. However, this second extension only adds "gauge copies" to the parameter space (see for example [22, 21] for an explanation). While type-I and type-III boundaries are "internal boundaries" of the M-theory moduli space, type-II contractions and the cubic cone lead to proper boundaries. At boundaries of type II the M-theory moduli space ends, and it has been shown that the tension of strings descending from M5-branes wrapped on the divisor goes to zero at such boundaries 40. Here the supergravity approximation breaks down, because infinitely many M-theory states become massless. Similarly, the supergravity approximation breaks down when approaching the cubic cone, and in this case no interpretation in terms of M-theory physics is known. $\S$

Using equation (3.5), for finite and non-zero Calabi-Yau volume $V$, we are able to infer regularity properties of the Kähler-cone metric $G$ from the matrix $M$ and vice versa: there is a one-to-one map of zero eigenvalues of $G$ to zero eigenvalues of $M$, i.e., if

$$
\left.\operatorname{det}\left(M_{I J}\right)\right|_{\mathcal{Y}^{\star} \rightarrow 0} \propto\left(\mathcal{Y}^{\star}\right)^{n},
$$

then there are $n$ linearly independent eigenvectors of $M$ (and of $G$ ) satisfying

$$
\left.v_{(i)}^{I} M_{I J}\right|_{\mathcal{Y}^{\star}=0}=0, \quad i=1 \ldots n .
$$

Here and in the following, $\mid \mathcal{Y}^{\star} \rightarrow 0$ denotes the limit approaching the boundary $\partial_{\star} \mathcal{K}$. Equation (3.7) is supposed to hold throughout the face $\partial_{\star} \mathcal{K}$. In particular, the null

$\S$ However, when dimensionally reducing on the M-theory cycle, such regions correspond to nongeometrical phases of type-IIA string theory on the same Calabi-Yau manifold 40, 41. 
The Kähler Cone as Cosmic Censor

Table 1. Behaviour of Kähler moduli-space metric at boundaries of the Kähler cone.

\begin{tabular}{cc}
\hline type of boundary & behaviour of $\operatorname{det}\left(G_{I J}\right)$ \\
\hline type I & regular \\
type II & zero \\
type III & regular \\
cubic cone & divergent \\
\hline
\end{tabular}

eigenvectors are determined by the triple intersection numbers, only. This implies that the components of the eigenvectors can be chosen to be integer. Hence, each zero eigenvector $v_{(i)}^{I}$ defines a divisor

$$
D_{(i)}:=v_{(i)}^{I} D_{I} \text {. }
$$

If there is a holomorphic surface within the homology class $D_{(i)}$, then its volume is given by

$$
\begin{aligned}
\frac{1}{2} v_{(i)}^{I} \int_{X} \omega_{I} \wedge J \wedge J & =\frac{1}{2} v_{(i)}^{I} c_{I \tilde{J} \tilde{K}} \mathcal{Y}^{\tilde{J}} \mathcal{Y}^{\tilde{K}}+v_{(i)}^{I} c_{I \tilde{J} \star} \mathcal{Y}^{\tilde{J}} \mathcal{Y}^{\star}+\frac{1}{2} v_{(i)}^{I} c_{I \star \star} \mathcal{Y}^{\star} \mathcal{Y}^{\star} \\
& =\left.v_{(i)}^{I} M_{I \tilde{J}}\right|_{\mathcal{Y}^{\star}=0} \mathcal{Y}^{\tilde{J}}+\left.2 v_{(i)}^{I} M_{I \star}\right|_{\mathcal{Y}^{\star}=0} \mathcal{Y}^{\star}+\frac{1}{2} v_{(i)}^{I} c_{I \star \star} \mathcal{Y}^{\star} \mathcal{Y}^{\star} \\
& =\frac{1}{2} v_{(i)}^{I} c_{I \star \star} \mathcal{Y}^{\star} \mathcal{Y}^{\star}
\end{aligned}
$$

where we have used equation (3.7). As a consequence, the divisors $D_{(i)}$, which are associated to null eigenvectors $v_{(i)}$, can never perform a type-III contraction, which is characterized by $\operatorname{Vol}(D) \propto \mathcal{Y}^{*}$. Irrespective of whether there exists a holomorphic surface in the class $D_{(i)}$, we learn that the moduli-space metric is always regular at boundaries of type I and type III. On the other hand, by definition of a type-II boundary ( $4 \rightarrow 0$ ", i.e. $\left.\operatorname{Vol}(D) \propto\left(\mathcal{Y}^{\star}\right)^{2}\right)$, we know that there exists at least one surface with homology class $D=v^{I} D_{I}$, which collapses to a point at $\partial_{\star} \mathcal{K}$. Hence the moduli-space metric develops a zero eigenvalue at boundaries of type II. At the cubic cone, the determinant of the moduli space generically diverges. More precisely, there are two cases: If $V \propto\left(\mathcal{Y}^{\star}\right)^{3}$, or $V \propto\left(\mathcal{Y}^{\star}\right)^{2}$, then the determinant of $G$ always diverges, see equation (3.5), since the determinant of the matrix $M$ can never compensate the zero in the denominator. What happens in the remaining case, $V \propto \mathcal{Y}^{\star}$ is that generically the determinant of $G$ blows up, while at special points $\operatorname{det} M$ can compensate the zero in the denominator of equation (3.5). Table [1 summarizes our result.

The generalisation of the proof to non-toric Calabi-Yau manifolds, where the global parameterisation of the Kähler moduli space which we have used above need not exist, is as follows. As in the proof above, at the cubic cone $W$ the volume of $X$ vanishes (i.e., $V=0$ ) and generically curvature singularities occur in domain wall solutions. Moreover, the Kähler cone metric also diverges at the cubic cone. Since the Kähler cone is locally 
polyhedral away from $W$, we know that for each of the primitive faces there exists a local parameterisation of the form (3.6). There can be accumulation points of faces, but these are known to reside inside the cubic cone [34]. Thus, the proof is valid for all Calabi-Yau three-folds.

Now we are able to interpret the singularities of section 2 in terms of M-theory physics. Singularities of type (i) correspond to the cubic cone where the volume of the Calabi-Yau three-fold goes to zero. Singularities of type (ii) can occur in two different situations: The first is that one has reached a type-II boundary (i.e., $\operatorname{det} G=0$ ). On these boundaries the internal manifold and the effective supergravity lagrangian become singular, and tensionless strings appear, as discussed above. However, there is also the possibility that a singularity of type (ii) arises because one has crossed a boundary of type I or type III before, so that one is outside the Kähler cone. This situation is analogous to the enhançon mechanism [17]. When reaching boundaries of type I or type III, the triple-intersection numbers and therefore the low-energy equations of motion and the space-time metric change. Continuation of domain-wall solutions through typeI boundaries have been considered in Ref. [28], whereas continuation of black-hole and black-string solutions through type-I and type-III boundaries have been studied in Ref. [21]. Here we only need to use that type-I and type-III boundaries are internal boundaries of the extended Kähler cone, and that the metric of the extended Kähler cone does not become singular. After crossing such boundaries the moduli take values in another Kähler cone, and there our proof of absence of naked singularities applies again. In conclusion we see that in M-theory singularities only occur on the boundary of the extended Kähler cone, where the internal manifold and the five-dimensional effective lagrangian become singular, and the description in terms of five-dimensional supergravity breaks down.

\subsection{Example: The $\mathcal{F}_{1}$-Model}

Here, we present a well known example of a Calabi-Yau manifold with $h^{1,1}=3$ [42, 43], which has all features discussed in the last subsections. It is an elliptic fibration over the first Hirzebruch surface $\mathcal{F}_{1}$. It turns out to be convenient to choose the following non-adapted parametrisation of the Kähler cone:\|

$$
\mathcal{K}=\left\{S, T, U \in \mathbb{R} \mid T>U>0, \quad S>\frac{T+U}{2}\right\}, \quad V=S T U+\frac{1}{3} U^{3} .
$$

The matrices $M_{I J}$ and $G_{I J}$ take the form

$M=\frac{1}{2}\left(\begin{array}{ccc}2 U & S & T \\ S & 0 & U \\ T & U & 0\end{array}\right), \quad G=\frac{1}{6 V^{2}}\left(\begin{array}{ccc}U^{4}+3 T^{2} S^{2} & 2 S U^{3} & 2 T U^{3} \\ 2 S U^{3} & 3 U^{2} S^{2} & -U^{4} \\ 2 T U^{3} & -U^{4} & 3 U^{2} T^{2}\end{array}\right)$

\|In this subsection, $U$ denotes one of the scalar fields, and not the function appearing in the space-time metric. 
The Kähler Cone as Cosmic Censor

Table 2. $\mathcal{F}_{1}$-model Kähler cone, cf. figure 1

\begin{tabular}{clll}
\hline location & name & physics & $\operatorname{det}\left(G_{I J}\right)$ \\
\hline$U=0$ & cubic cone & unknown & divergent \\
$T=U$ & type I & flop transition & regular \\
$S=\frac{T+U}{2}$ & type III & $S U(2)$ symmetry enhancement & regular \\
$S T=U^{2}$ & discriminant line & unphysical & degenerate \\
\hline
\end{tabular}

with determinants

$$
\operatorname{det} M=\frac{U}{4}\left(S T-U^{2}\right), \quad \operatorname{det} G=\frac{\operatorname{det} M}{2 V^{3}},
$$

satisfying equation (3.5). Figure 1 displays the Kähler cone of this model and table 2 summarises the information in figure 1 [ [13, 22, 21]. Note that the curves with $\operatorname{det} G=\infty$ $(U=0)$ and with $\operatorname{det} G=0\left(S T=U^{2}\right)$, lie always outside or at boundaries of $\mathcal{K}$, in accord with the general statement of table $\mathbb{1}$. The line $S T=U^{2}$ is called discriminant line, because $S T-U^{2}$ is the discriminant of a specific polynomial, whose zeros are in one-to-one corresponce with diverging derivatives of the scalar fields [21]. As long as $S T>U^{2}$, this polynomial does not have real zeros, and derivatives of scalar fields cannot diverge. Observe that the discriminant line lies beyond the type-III boundary, where gauge symmetry is enhanced. Therefore the naked space-time singularities occurring at $S T=U^{2}$ are unphysical [18, 21]. For the analogous black hole solution, the correct non-singular continuation beyond the type-III boundary is described in [21].

The extended Kähler cone of the model is obtained by extending it along the flop

line, $S=(T+U) / 2$. The flopped image of $\mathcal{K}$ has boundaries of type II, where the metric degenerates.

\section{Electric BPS Solutions of Ungauged Five-dimensional Supergravity}

Let us now indicate how our analysis can be adapted to electric BPS solutions, which, when no naked space-time singularity occurs, are black holes. Here we consider a different five-dimensional supergravity theory, namely ungauged supergravity with an arbitrary number of vector and hypermultiplets, which admits five-dimensional Minkowski space as a fully supersymmetric solution. Since hypermultiplets are trivial in electric BPS solutions, we can ignore them and use the general vector multiplet lagrangian of [24]. Again this sector is completely determined by a real cubic prepotential. Five-dimensional ungauged supergravity with vector and hypermultiplets can be obtained by dimensional reduction of M-theory on a Calabi-Yau three-fold without flux [44, 45]. As before, the vector multiplet scalars parametrise a cubic hypersurface in the Kähler cone, and the coefficients $c_{I J K}$ are the triple-intersection numbers. 


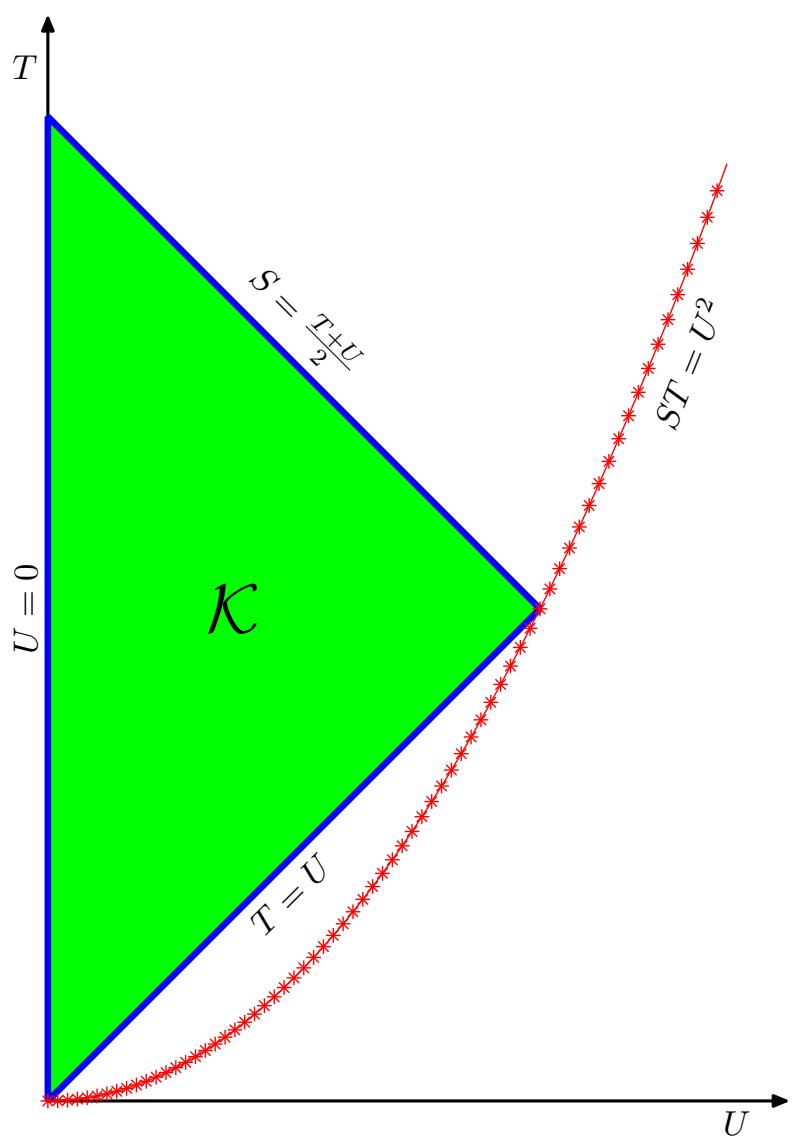

Figure 1. Section of the $\mathcal{F}_{1}$-model Kähler cone for fixed modulus $S>0$.

Electric BPS solutions of ungauged five-dimensional supergravity have been found in [46] (who use conventions slightly different from those of [24]). The line element is

$$
\mathrm{d} s^{2}=-\exp [-4 U(r)] \mathrm{d} t^{2}+\exp [2 U(r)]\left\{\mathrm{d} r^{2}+r^{2} \mathrm{~d} \Omega_{(3)}^{2}\right\},
$$

and the non-vanishing components of the field strengths take the form

$$
F_{t r}^{I}=-\partial_{r}\left(\exp [-3 U(r)] Y^{I}(r)\right) .
$$

Here $Y^{I}(r)=\exp [U(r)] X^{I}(r)=\exp [U(r)] V^{-1 / 3} \mathcal{Y}^{I}$ are again rescaled scalar fields, subject to the condition $\mathcal{V}(Y)=\exp [3 U(r)]$. The full set of equations of motion and Killing spinor equations reduces to the same set of algebraic equations as for domain walls $(c . f .(2.5))$ :

$$
c_{I J K} Y^{J}(r) Y^{K}(r)=2 H_{I}(r),
$$

where this time the functions $H_{I}(r)$ are harmonic with respect to four-dimensional space. For the above line element, which describes a single-centered solution, these take the form

$$
H_{I}(r)=c_{I}+\frac{q_{I}}{r^{2}}
$$


where $r$ is the radial coordinate, $q_{I}$ are the electric charges carried by the solution, and $c_{I}$ determine the values of the moduli at infinity. It is remarkable that two different spacetime geometries, which arise as solutions of two different supergravities, are governed by the same dynamical system on their vector multiplet manifolds (which agree, if $c_{I J K}$ are the same). This becomes more transparent through dimensional reduction to $0+1$ dimensions 447.

The Ricci scalar corresponding to the above line element takes the form

$$
R=-2 r^{-1} \exp [-2 U]\left(r U^{\prime \prime}+3 r\left(U^{\prime}\right)^{2}+3 U^{\prime}\right) .
$$

In the coordinates we have chosen, the solution becomes asymptotically flat for $r \rightarrow \infty$, whereas $r=0$ is either the event horizon of a black hole, or a singularity. The limit $r \rightarrow 0$ is related to the celebrated black-hole attractor mechanism, which was first discovered in four-dimensional extended supersymmetry [48], but also occurs in fivedimensional supergravity [46]. BPS solutions of supergravities with eight supercharges have four Killing spinors, but if one imposes that they behave regularly at $r=0$, then the number of Killing spinors must double in the limit $r \rightarrow 0$. This implies that the solutions interpolate between two fully supersymmetric solutions, flat space at $r \rightarrow \infty$, and $A d S^{2} \times S^{2}$ or $A d S^{2} \times S^{3}$ at $r \rightarrow 0$, depending on whether the total number of space-time dimensions is four or five. These geometries describe the event horizons of black holes, which are generalisations of the extreme Reissner-Nordström solution. Moreover, the scalar fields take discrete fixed point values at the horizon, which are determined by the so-called stabilisation equations. The attractor mechanism is crucial for understanding black-hole entropy along the lines of [49], because it implies that the Bekenstein-Hawking entropy does not change when varying the values of scalar fields at infinity. While the original work of 48 focused on the behaviour of four-dimensional black holes close to their horizon, it soon became clear that the corresponding full black-hole solutions are determined by a rescaled version of the stabilisation equations, which have been called generalised stabilisation equations [50]. Equation (4.1) is the five-dimensional version of the generalised stabilisation equation, and the stabilisation equation determining the geometry at $r \rightarrow 0$ can be found by a suitable scaling limit [46]. In four dimensions it has been shown that the generalised stabilisation equations are not only sufficient, but also necessary for having a supersymmetric solution [51]. It was also shown that one cannot switch on non-trivial hypermultiplets, but that it is possible to include the effect of certain higher curvature terms [51]. We refer to [52] for a review. These results should survive in the de-compactification limit and therefore also apply to five-dimensional black holes.

Note that the supersymmetric attractor mechanism, which we just reviewed, does not occur in the domain-wall solutions of Hořava-Witten theory. The reason is that the five-dimensional bulk supergravity theory does not have fully supersymmetric solutions, due to the runaway of the volume modulus [19, 20, 27]. Therefore these 
domain walls do not interpolate between vacua, but have to be cut by introducing boundaries. Even though there is no fixed-point behaviour in these solutions, they are nevertheless determined by the generalised stabilisation equations (2.5). There are other five-dimensional gauged supergravities, in particular those obtained by gauging the Rsymmetries of vector multiplets [25], which have domain walls interpolating different AdS vacua. For those the attractor mechanism applies, as pointed out in [30]. However, it is not known how to obtain these supergravities as compactifications of string or M-theory. One interesting speculation is that the run-away behaviour of the volume in Hořava-Witten domain walls stops, once a minimal volume of the Calabi-Yau space has been reached [27]. This has found some support recently by new results about loop corrections to the universal hypermultiplet 53.

Let us now consider the question of naked singularities in electric BPS solutions. Since the behaviour at $r=0$ is taken care of by the attractor mechanism, we only need to analyse under which conditions a solution can develop a naked singularity for finite values $r=r_{S}, \infty>r_{S}>0$ of the radial variable. As for domain walls, the solution is determined by a flow on the vector-multiplet manifold, which is now parametrised by $r, \infty>r>0$, instead of $y$. Whereas the parameters $c_{I}$ determine the behaviour for $r \rightarrow \infty$, the parameters $q_{I}$ determine it for $r \rightarrow 0$. By inspection of the Ricci scalar, and of other curvature invariants, a curvature singularity occurs if and only if either $U=0$, or if $U^{\prime}$ or $U^{\prime \prime}$ diverge at $r=r_{S}$. Since $r>0$, the harmonic functions $H_{I}$ and all their derivatives are finite, and the analysis done for domain walls goes through. Thus we find again that in supergravity regular and singular solutions are equally generic, while solutions in Calabi-Yau compactifications cannot become singular, as long as the scalar fields take values inside the extended Kähler cone. Singularities do occur when the moduli reach the boundary of the extended Kähler cone.

For completeness, let us finally mention further details which are different from the case of domain walls, but fortunately do not interfere with our analysis. These differences occur because in ungauged supergravity vector and hypermultiplets only couple through gravity, while there are gauge couplings and a scalar potential in gauged supergravity. As we have already mentioned the hypermultiplets are trivial in electric BPS solutions. Since the overall volume of the internal space sits in a hypermultiplet, this implies that it is constant, in contrast to the domain-wall case, where it is a specific function of the vector-multiplet moduli (2.4). This has some impact on the discussion of the cubic cone, where the Calabi-Yau volume goes to zero. While this locus can be approached in domain-wall solutions, it cannot in black-hole solutions, because the volume is fixed. Nevertheless, there is a related degeneration occurring in the black-hole case, where some cycles go to zero while other go to infinity, in such a way that the total volume is constant. This happens for example at one boundary of the Kähler cone of the $\mathcal{F}_{1}$-model [21]. Another minor difference is that, as we have seen in section 2, for domain walls one can prove that $U^{\prime}$ cannot diverge if $U$ is finite. The difference is that 
derivatives of the linear functions $H_{I}(y)$ are constant or vanishing, while the derivatives of the functions $H_{I}(r)$ are not constant, though still finite. As a consequence, in the black-hole case $U^{\prime}$ can become singular for finite $U$, but this can only happen outside the extended Kähler cone, or at points on its boundary.

\section{Conclusions}

We have analysed domain-wall solutions (2.3) of five-dimensional gauged supergravity theories. Our analysis applies, with only minor modifications, to black-hole solutions of ungauged supergravity. In particular, we have investigated the appearance of curvature singularities in domain-wall solutions and found that there are two possible causes, namely $V \rightarrow 0$ and $\left|V^{\prime \prime}\right| \rightarrow \infty$. Within a pure supergravity perspective this is all what can be achieved.

Embedding these theories into a higher-dimensional theory, i.e., eleven-dimensional supergravity on a Calabi-Yau flux background, changes the situation: the fivedimensional scalar fields become identified with volumes of 2-cycles in the Calabi-Yau three-fold. Therefore, they are required to take values inside the Kähler cone of the Calabi-Yau manifold.

We have proven that naked curvature singularities cannot occur as long as the scalars take values inside the extended Kähler cone. Singularities do appear when the scalars reach the boundary, where the internal manifold and the five-dimensional effective supergravity action become singular, and where one needs to understand new M-theory physics, such as tensionless strings.

Furthermore, we have obtained model-independent information on the behaviour of the Kähler moduli-space metric near boundaries of the Kähler cone. We have proven that the moduli-space metric is regular at boundaries of type I and of type III, whereas it develops zero eigenvalues at boundaries of type II and diverges at the cubic cone, see table 1 .

Our result is consistent with the fact that one can extend the Kähler cone at boundaries with type-I and type-III contractions by gluing the Kähler cone of a different Calabi-Yau manifold to the corresponding face [40, 15, 22]. Moreover, it complements the other important model-independent result about black holes in CalabiYau compactifications of M-theory, which states that attractor points are unique in the extended Kähler cone [54].

Results about other geometries support the idea that the enhançon-like mechanism, established in this paper for domain walls and electric BPS solutions, is much more generic. In particular, ungauged five-dimensional supergravity also has magnetic BPS solutions [46]. For these it is very simple to show that they cannot have naked singularities as long as the moduli take values in the extended Kähler cone [21]. Moreover, it was observed that cosmological solutions of Kasner type do not become 
singular, as long as the moduli take values in the extended Kähler cone [23].

A natural next step is trying to establish a similar mechanism for black holes of fourdimensional $\mathcal{N}=2$ supergravity. Since in this case one has to deal with a holomorphic instead of a real cubic prepotential, this will require a non-trivial extension of the framework used in this paper. When considering type-IIA string theory on a Calabi-Yau three-fold, which is the dimensional reduction of the M-theory setup employed in this paper, then the moduli space is a complexified version of the Kähler cone. Now the overall volume $V$ sits in a vector multiplet, and the metric on the Kähler cone gets $\alpha^{\prime}$ corrections. Moreover, the former boundaries of the Kähler cone now become so-called non-geometric phases, which are not described by Calabi-Yau sigma models, but by other types of world-sheet conformal field theories [41, 40]. This is a technical complication, but a physical bonus, because now the cubic cone is on the same footing as the other degenerations. For example, the Kähler cone of the $\mathcal{F}_{1}$-model has a boundary which belongs to the cubic cone [21]. After dimensional reduction, this region corresponds to a Landau-Ginsburg theory. Moreover, one can use mirror symmetry to study IIB string theory on the mirror Calabi-Yau manifold instead. In this description the relevant scalar manifold is the moduli space of complex structures, which does neither get $\alpha^{\prime}$ nor string loop corrections. Thus the best strategy is to investigate the links between complexstructure moduli spaces of Calabi-Yau spaces and BPS space-time geometries. This is a vast and very interesting subject, and some of its aspects have already been explored [55, 56]. Singularities of the complex moduli space occur in complex co-dimension one, only. Therefore they will be avoided by generic solutions. Many of the singularities in co-dimension one and higher have known physical interpretations in terms of additional massless states coming from wrapped D-branes. Since D-branes can be treated by world-sheet techniques, one now has additional tools besides effective supergravity. In particular, the best strategy to understand the M-theory physics of the cubic cone and of tensionless strings is the dimensional reduction to type II string theory.

A point which deserves further investigation is the relation of supergravity actions to string compactification on singular Calabi-Yau manifolds. Two different questions can be investigated in this context: First, we would like to know what are the supergravity lagrangians corresponding to the " $4 \rightarrow 0$ " boundary theories, i.e., which supergravity lagrangians correspond to the non-geometric phases. And secondly, it would be interesting to use the supergravity description in order to learn about the cohomology and intersection homology of the singular Calabi-Yau three-folds obtained by type I, II and III contractions.

ฯ We thank Albrecht Klemm for pointing this out. 


\section{Acknowledgments}

This work is supported by the 'Schwerpunktprogramm Stringtheorie' of the DFG.

\section{References}

[1] Penrose R 1965 Phys. Rev. Lett. 1457

Hawking S W 1967 Proc. R. Soc. Lond. A 300182

Hawking S W and Penrose R 1970 Proc. R. Soc. Lond. A 314529

[2] Penrose R 1979, "Singularities and Time Asymmetry" in: General Relativity, an Einstein Centenary Survey ed. Hawking S W and Israel W (Cambrigde University Press)

[3] Wald R M 1984 General Relativity (Chicago: University of Chicago Press)

[4] Kallosh R, Linde A, Ortin T, Peet A and Van Proeyen A 1992 Preprint hep-th/9205027

[5] Gibbons G W and Hull C M 1982 Phys. Lett. B 109190

Tod K P 1983 Phys. Lett. B 121241

[6] Giveon A, Rabinovici E and Sever A 2003 Fortsch. Phys. 51805

[7] Dixon L, Harvey J, Vafa C and Witten E 1985 Nucl. Phys. B 261678

Dixon L, Harvey J, Vafa C and Witten E 1986 Nucl. Phys. B 274285

[8] Horowitz G T and Steif A R 1991 Phys. Lett. B 25891

[9] Horowitz G T and Polchinski J 2002 Phys. Rev. D 66103512

[10] Liu H, Moore G and Seiberg N 2002 JHEP 0206045

Liu H, Moore G and Seiberg N 2002 JHEP 0210031

[11] Cornalba L and Costa M S 2003 Preprint hep-th/0310099

[12] Aspinwall P S, Greene B R and Morrison D R 1994 Nucl. Phys. B 416414

[13] Strominger A 1995 Nucl. Phys. B 4519

[14] Greene B R, Morrison D R and Strominger A 1995 Nucl. Phys. B 451109

[15] Katz S, Morrison D R and Plesser M R 1996 Nucl. Phys. B 477105

[16] Klemm A and Mayr P 1996 Nucl. Phys. B 46937

[17] Johnson C V, Peet A and Polchinski J 2000 Phys. Rev. D 61086001 Johnson C V, Myers R C, Peet A and Ross S F 2001 Phys. Rev. D 64106001

[18] Kallosh R, Mohaupt T and Shmakova M 2001 J. Math. Phys. 423071

[19] Lukas A, Ovrut B A, Stelle K S and Waldram D 1999 Phys. Rev. D 59086001

[20] Lukas A, Ovrut B A, Stelle K S and Waldram D 1999 Nucl. Phys. B 552246

[21] Mohaupt T 2003 Fortsch. Phys. 51787

[22] Gaida I, Mahapatra S, Mohaupt T and Sabra W A 1999 Class. Quantum Grav. 16416

Mohaupt T and Zagermann M 2001 JHEP 0112026

Louis J, Mohaupt T and Zagermann M 2003 JHEP 0302053

Järv L, Mohaupt T and Saueressig F 2003 Preprint hep-th/0310173

[23] Järv L, Mohaupt T and Saueressig F 2003 Preprint hep-th/0310174

Järv L, Mohaupt T and Saueressig F 2003 Preprint hep-th/0311016

[24] Gunaydin M, Sierra G and Townsend P K 1984 Nucl. Phys. B 242244

[25] Gunaydin M, Sierra G and Townsend P K 1985 Nucl. Phys. B 253573

[26] Ceresole A and Dall'Agata G 2000 Nucl. Phys. B 585143

[27] Behrndt K and Gukov S 2000 Nucl. Phys. B 580225

[28] Greene B R, Schalm K and Shiu G 2001 J. Math. Phys. 423171

[29] de Wit B and Van Proeyen A 1992 Commun. Math. Phys. 149307

[30] Bergshoeff E, Kallosh R and Van Proeyen A 2000 JHEP 0010033

[31] Witten E 1996 Nucl. Phys. B 471135 
[32] Gurrieri S, Louis J, Micu A and Waldram D 2003 Nucl. Phys. B 65461

[33] Witten E 1997 J. Geom. Phys. 221

[34] Wilson P M H 1992 Invent. math. 107561

Wilson P M H 1993 Invent. math. 114231

[35] Oda T 1988 Convex Bodies and Algebraic Geometry (Berlin/Heidelberg: Springer)

[36] Reid M 1983 Decomposition of Toric Morphisms in: Arithmetic and Geometry vol II Progr. Math. 36 (Boston, MA: Birkhäuser) pp 395

[37] Fujino O and Sato H 2003 Preprint math.AG/030718

[38] Strominger A 1985 Phys. Rev. Lett. 552547

[39] Bodner M, Cadavid A C and Ferrara S 1991 Class. Quantum Grav. 8789

[40] Witten E 1996 Nucl. Phys. B 471195

[41] Witten E 1993 Nucl. Phys. B 403159

[42] Louis J, Sonnenschein J, Theisen S and Yankielowicz S 1996 Nucl. Phys. B 480185

[43] Chou A, Kallosh R, Rahmfeld J, Rey S J, Shmakova M and Wong W K 1997 Nucl. Phys. B 508147

[44] Cadavid A C, Ceresole A, D'Auria R and Ferrara S 1995 Phys. Lett. B 35776

Papadopoulos G and Townsend P K 1995 Phys. Lett. B 357300

[45] Ferrara S, Khuri R R and Minasian R 1996 Phys. Lett. B 37581

[46] Chamseddine A, Ferrara S, Gibbons G W and Kallosh R 1997 Phys. Rev. D 553647

Sabra W A 1998 Mod. Phys. Lett. A 13239

Chamseddine A H and Sabra W A 1999 Phys. Lett. B 46063

[47] Behrndt K, Gukov S and Shmakova M 2001 Nucl. Phys. B 60149

[48] Ferrara S, Kallosh R and Strominger A 1995 Phys. Rev. D 525412

Strominger A 1996 Phys. Lett. B 38339

Ferrara S and Kallosh R 1996 Phys. Rev. D 541514

Ferrara S and Kallosh R 1996 Phys. Rev. D 541525

[49] Strominger A and Vafa C 1996 Phys. Lett. B 37999

[50] Sabra W A 1997 Mod. Phys. Lett. A 122585

Sabra W A 1998 Nucl. Phys. B 510247

Behrndt K, Lüst D and Sabra W A 1998 Nucl. Phys. B 510264

[51] Cardoso G L, de Wit B, Käppeli J and Mohaupt T 2000 JHEP 0012019

[52] Mohaupt T 2001 Fortsch. Phys. 493

[53] Antoniadis I, Minasian R, Theisen S and Vanhove P 2003 Class. Quantum Grav. 205079

[54] Wijnholt M and Zhukov S 1999 Preprint hep-th/9912002

[55] Moore G W 1998 Preprint hep-th/9807087

Moore G W 1998 Preprint hep-th/9807056

[56] Denef F 1999 Nucl. Phys. B 547201

Denef F 2000 JHEP 0008050

Denef F, Greene B R and Raugas M 2001 JHEP 0105012 